\title{
Methylene Blue as a Diagnostic Aid in the Early Detection of Potentially Malignant and Malignant Lesions of Oral Mucosa
}

\author{
Abraham Lejoy ${ }^{1}$, Rai Arpita ${ }^{2}$, Burde Krishna ${ }^{1}$, Naikmasur Venkatesh ${ }^{1}$
}

ABSTRACT

BACKGROUND: In vivo stains are the prompt resources, which have emerged in recent years to aid as clinical diagnostic tools in detecting early potentially malignant and malignant lesions. Toluidine blue, by its property of retaining in the increased DNA and RNA cellular activity areas, aids in delineating the suspicious areas. However, it is hazardous if swallowed, and has been shown to have toxicity to fibroblasts. Methylene blue has a similar chemical structure and exhibits similar physicochemical properties as toluidine blue. It is less toxic to the human body and has recently been proposed for screening some gastrointestinal or prostate tumors. The application of this material in detecting oral lesions has so far not been addressed. The objective of this study was to evaluate the sensitivity and reliability of in vivo staining with methylene blue as a diagnostic adjunct in screening for oral malignant or potentially malignant lesions.

METHODS: The present study involved the examination of 75 patients suspected of having oral malignant or potentially malignant lesions by methylene blue staining. The results of methylene blue uptake were compared with a simultaneous biopsy of these lesions.

RESULTS: The overall sensitivity was 95\% (100\% for malignancy and $92 \%$ for potentially malignant lesions) and specificity was 70\%. The positive predictive value was $91 \%$ and negative predictive value of $80 \%$ was observed in the study.

CONCLUSION: We consider that methylene blue staining is a useful diagnostic adjunct in a large, community-based oral cancer screening program for high-risk individuals.

KEYWORDS: Methylene blue, Oral malignant, Potentially malignant

DOI: http://dx.doi.org/10.4314/ejhs.v26i3.2

\section{INTRODUCTION}

Oral cancer is one of the most frequently observed malignancies in the world and shows marked geographic differences in occurrence. Oral cancer is common where betel quid chewing, bidi smoking, alcohol and tobacco consumption are high. Thus, it is frequently observed in South East Asia where more than 100,000 new cases are reported every year (1).

There are about 700,000 new cases of cancers every year in India out of which tobacco related cancers are about 300,000 . The cost of treatment of oral cancer is about 3.5 lakh. This can be completely prevented by simple changes in lifestyle and regular screening. About 2000 deaths a day in India is tobacco related (2).

Early detection of oral carcinoma is of paramount importance as it can best be cured at its earliest stage. Several studies have shown that patient mortality reduces considerably when oral carcinoma is treated at initial stage (3). Similarly, it is equally important to detect and treat potentially malignant lesions as their progress to malignancy can be prevented (4).

\footnotetext{
${ }^{1}$ SDM College of Dental Sciences and Hospital, Dharwad

${ }^{2}$ Department of Oral Medicine and Radiology, Faculty of Dentistry, Jamia Millia Islamia, New Delhi

Corresponding Author: Rai Arpita, Email: arpitadoc@gmail.com

Among the diagnostic tools, in vivo staining is advocated as a simple, inexpensive, and fairly sensitive method (4). Periodic clinical examination
} 
by doing vital staining reduces the mortality rate by $32 \%$ in high risk individuals (4). Adjunctive aids such as Toluidine blue (tolonium chloride) have been widely accepted in large scale screening for oral cancer diagnosis (5-10). The disadvantages of Toluidine blue are its hazardous effect if swallowed and its reported toxicity to fibroblasts (4).

Methylene blue has a similar chemical structure and exhibits similar physicochemical properties to Toluidine blue. It is less toxic to the human body and recently been proposed for screening some gastrointestinal, prostate and bladder tumors (11-13). The application of this material in detecting oral lesions has not been extensively addressed. Hence, this study was undertaken to highlight the efficacy of Methylene Blue staining in the early detection of potentially malignant and malignant lesions of the oral mucosa.

\section{MATERIALS AND METHODS}

Subjects: The efficacy of $1 \%$ methylene blue in the detection of potentially malignant and malignant oral lesions/condition was evaluated among a group of 125 patients at risk for oral malignancy. The subjects were divided into two groups:

Cases: Seventy-five patients with clinical suspicions of oral potentially malignant and malignant lesions were selected at random from the Department of Oral Medicine and Radiology for the study group. The patients did not have previous history of oral cancer and had not undergone any previous treatment for oral malignancy.

Controls: Ffty controls without the habits of tobacco in any form (smoking and quid chewing) were included in our control study.

The clinical data regarding the patients' gender, age and habits of tobacco use were collected. All the patients were subjected to a systematic oral examination for clinical diagnosis which was categorized clinically into the following 4 groups:

Group 1: Homogeneous leukoplakia, i.e. white, uniform, flat lesion with a smooth, wrinkled, or corrugated surface, not able to be scraped

Group 2: Nonhomogeneous leukoplakia-white lesion with irregular and exophytic surface
Group 3: Erythroplakia-red lesion with ill-defined margin

Group 4: Carcinoma-localized and superficial ulcero-proliferative lesions.

Subjects in the study as well as in control groups were subjected to methylene blue staining procedure.

Formulation of tissue stain: Methylene blue dye system had two solution bottles. The dye rinse solution (Bottle A) had 1\% methylene blue, 1\% malachite, $0.5 \%$ eosin, glycerol and dimethylsulfoxide. Pre- and post-rinse solution (Bottle B) had 1\% lactic acid and purified water.

Staining protocol: The oral cavity was examined and location, size, morphology and surface characteristics of suspect lesions were recorded and photographed. All patients rinsed their mouths with 1\% lactic acid (Bottle B) for 20 seconds to remove food debris and excess saliva and to provide a consistent oral environment. The mucosa of the target area was gently dried with gauze to ensure that the lesion was not contaminated with saliva. For equivocal staining, $1 \%$ methylene blue dye (Bottle A) was applied directly over the suspected area with the help of cotton and kept in place for 20 seconds.

Patients then rinsed their mouths again with $1 \%$ lactic acid (Bottle B) for 20 seconds to wash out the excess dye. The pattern of dye retention was assessed by the intensity of stain retention on the lesion:

- Local, stippled, patchy and deep blue stains

- Wide, shallow or faint blue stains

For equivocal staining, 1\% lactic acid (Bottle B) solution was applied with cotton rolls to wipe out the staining surface. If the blue stain was washed out, negative reaction was recorded and vice versa. If the patient had a highly suspicious lesion that was not stained by the solution, he/she was instructed to revisit within 14 days to repeat the test in order to reduce the false-negative rate.

Interpretation of stain: The staining pattern observed was divided into 3 categories:

Dark uptake: Cases where the lesion or portion of the lesion stains dark blue in a solid or stippled fashion

Light uptake: Well circumscribed light blue staining pattern with intensity of stain lower than lesions showing dark uptake 
No uptake/ Equivocal staining: Cases where there are areas of mucosa which stains with a faint blue color

The results of methylene blue dye staining were recorded with photographs. Incisional/excisional biopsy was performed simultaneously in the suspected lesions to compare the accuracy of the diagnostic capability of methylene blue.

Histologic examination: For the subjects in the study group which consisted of 75 patients, a punch of biopsy was performed in the most obvious staining area of the suspicious lesion of patients under local anaesthesia. If there was no dye uptake in the lesions, the biopsy specimen was taken from the area judged by a specialist's experience. The specimens were then fixed in $10 \%$ formalin and processed in the pathology laboratory for initial routine pathologic diagnosis. Thus, the false positives, the false negatives, the sensitivity and the specificity were calculated. Biopsy was not performed on subjects in the control group without dye uptake considering ethical issues.

The dye uptake results were correlated with the histopathological report in order to estimate the false positives, false negatives, the sensitivity and the specificity rates. All the specimens were microscopically evaluated by pathologists who were blind to the results of methylene blue stain. The pathology reports of the lesions were classified as:

(1) Dysplastic lesions which were further subcategorized as mild, moderate and severe dysplasia
(2) Non-dysplastic lesions including epithelial hyperplasia, lichen planus and hyperkeratosis

(3) Malignant lesions including verrucous carcinoma and oral squamous cell carcinoma.

Statistical analysis: Statistical analysis was performed, including sensitivity, specificity, positive and negative predictive values. The association of methylene blue uptake and pathologic diagnosis was analyzed using Yates corrected chi-square test. A p value (probability value) of less than 0.01 was considered very significant.

\section{RESULTS}

Subject characteristics: One hundred and twenty- five subjects ( 75 patients and 50 volunteer students) were enrolled in this study. The patients' age (patient group) ranged from 21 to 73 years (mean age 51.3 years), with the male to female ratio being 14:1. The student's ages (control group) ranged from 19 to 25 years (mean age 22 years).

The oral mucosal lesions selected in this study were divided clinically into four categories homogenous leukoplakia in 39 patients, nonhomogenous leukoplakia in 27 patients, oral carcinoma in 4 and erythroplakia in 5. The results of the present study showed three types of staining patterns with respect to the clinical diagnosis of oral mucosal lesions (Table 1).

Table 1: Methylene blue dye uptake

\begin{tabular}{lccccc}
\hline Uptake Pattern & HL $^{*}$ & NHL $^{\bullet}$ & Carcinoma & Erythroplakia & Number of Lesions \\
\hline Dark uptake & 0 & 39 & 5 & 4 & 48 \\
Light uptake & 12 & 0 & 0 & 0 & 12 \\
No uptake/equivocal staining & 15 & 0 & 0 & 0 & 15 \\
Total & $\mathbf{2 7}$ & $\mathbf{3 9}$ & $\mathbf{5}$ & $\mathbf{4}$ & $\mathbf{7 5}$ \\
\hline
\end{tabular}

*HL - Homogenous Leukoplakia ${ }^{\bullet}$ NHL- Non Homogenous Leukoplakia

Methylene blue staining related to the grade of pathology: The histopathological diagnosis of oral lesions and the results of staining are shown in Table 2. The pathologic grade was classified as absence of dysplasia (mild, moderate and severe dysplasia) and OSCC as previously described in the methods section. The cases which showed dark blue uptake of the methylene blue dye were diagnosed histologically as mild dysplasia in 5 patients and moderate to severe dysplasia and oral squamous cell carcinoma in 41 patients (Figure 1). The cases which showed light uptake clinically were diagnosed histopathologically as nondysplastic on 5 instances and mild dysplasia in 12 
cases (Figure 2). There were 12 cases which showed equivocal uptake and were histologically proven as non-dysplastic oral mucosal lesions (Figure 3).

Table 2: Staining pattern related to grade of pathology

\begin{tabular}{lllll}
\hline & $\begin{array}{l}\text { Dark } \\
\text { Uptake }\end{array}$ & Light Uptake & $\begin{array}{l}\text { No uptake or Equivocal } \\
\text { Stain }\end{array}$ & TOTAL \\
\hline No dysplasia & 0 & 5 & 12 & 17 \\
Mild dysplasia & 5 & 12 & 0 & 17 \\
Moderate /severe dysplasia/ OSCC & 41 & 0 & 0 & 41 \\
Total & $\mathbf{4 6}$ & $\mathbf{1 7}$ & $\mathbf{1 2}$ & $\mathbf{7 5}$ \\
\hline
\end{tabular}

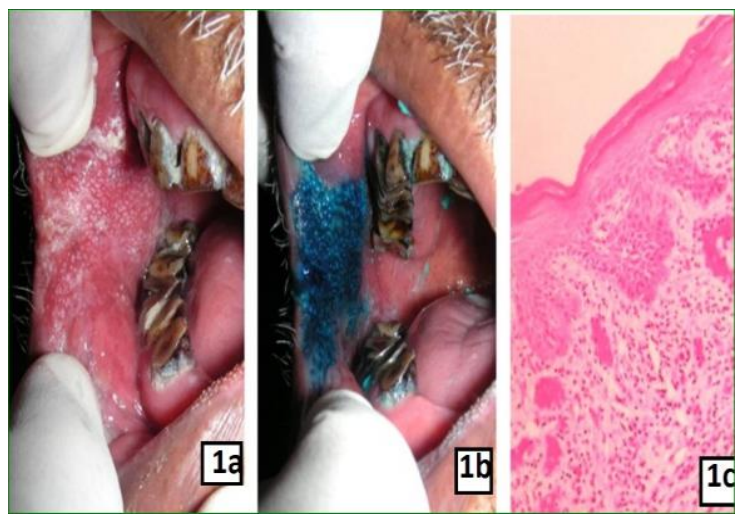

la: Clinical photograph showing nonhomogenous leukoplakia on right buccal mucosa 1b: Clinical photograph showing dark uptake of stain after application of methylene blue dye

1c: Photomicrograh showing moderate dysplasia (X10)

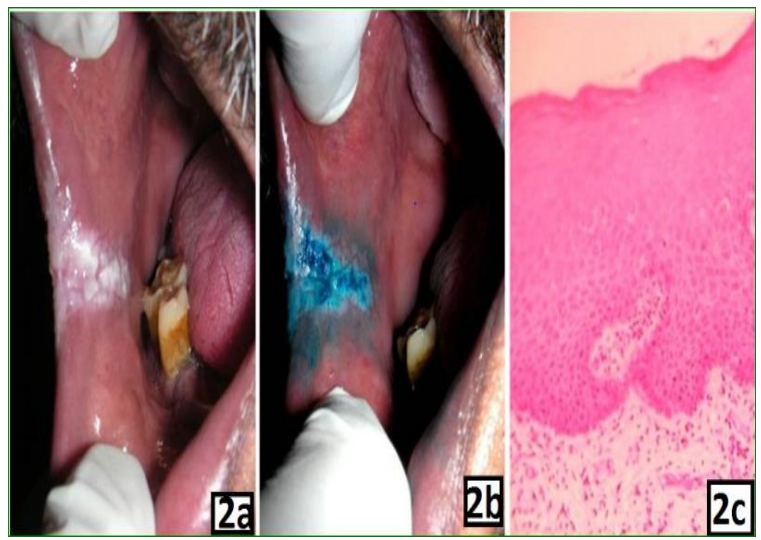

2a: Clinical photograph showing homogenous leukoplakia on right buccal mucosa

2b: Clinical photograph showing light uptake of stain after application of methylene blue dye 2c: Photomicrograh showing mild dysplasia (X100)

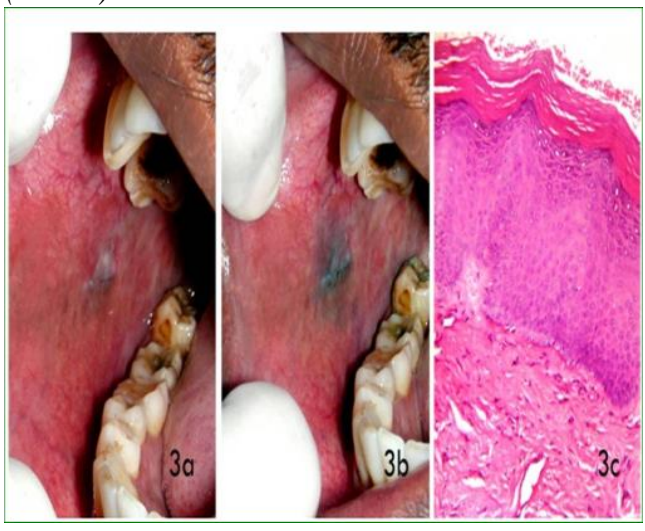

3a: Clinical photograph showing homogenous leukoplakia on right buccal mucosa

3b: Clinical photograph showing very mild uptake of stain or equivocal staining after application of methylene blue dye

3c: Photomicrograh showing no dysplastic features (X100)

In this study, 55 of 58 pathologically proven oral malignant or potentially malignant lesions were positive with deep and focal methylene blue staining. The overall sensitivity was $95 \%$. The three false negative cases were homogeneous leukoplakia on the buccal mucosa with histopathological diagnosis showing no dysplastic features. These lesions were stained with a faint blue colour.

In the study, 12 of 15 benign lesions showed negative staining. Thus, the specificity was $70 \%$. The results of staining with methylene blue for all lesions correlated well with the pathologic diagnosis as summarized in Table 3. Yates corrected chi-square showed significant 
differences among cancer/precancerous lesions, benign lesions and normal control groups ( $p<$ 0.01 ). Overall, the positive predictive value was
$91 \%(55 / 60)$, and the false predictive value was $80 \%(12 / 15)$.

Table 3. Efficacy of methylene blue application in pathologically proved cancer/precancerous lesion

\begin{tabular}{llll}
\hline Histological Diagnosis & Positive (\%) & Negative $(\%)$ & P Value \\
\hline OSCC/ Dysplasia $(\mathrm{n}=58)$ & $55(95)^{*}$ & $3(5)$ & $\mathrm{P}=0.0000(\mathrm{p}<0.001)$ \\
No Dysplasia $(\mathrm{n}=17)$ & $5(29)$ & $12(70)^{\bullet}$ & $\mathrm{P}=0.0010(\mathrm{P}<0.01)$ \\
Control $(\mathrm{n}=50)$ & $0(0)$ & $50(100)$ & \\
\hline
\end{tabular}

*Sensitivity; ${ }^{\bullet}$ Specificity

\section{DISCUSSION}

Oral cancer is very common in Southeast Asia, including India. Areca nut chewing, tobacco and alcohol consumption are the main etiologic factors inducing carcinogenesis in oral mucosa. Individuals with all the habits of smoking, alcohol consumption and areca nut chewing were reported to have 123 times the risk of people without such habits (14). Thus, oral cancer screening of highrisk individuals is very important in these countries.

For a large-scale community screening, certain dye materials help to identify abnormal mucosa tissue and raises oral examiners attention. The patients with suspicious lesions may be referred to oral physicians for further examinations.

The concept of two-step process of cancer development in the oral mucosa, i.e., the initial presence of a precursor (pre-cancerous) lesion subsequently developing into cancer is well established and the early detection of oral mucosal epithelial dysplasias could potentially halt the progression of these lesions into malignant transformation $(15,16)$. Thus, establishment of useful and objective techniques adjunctive to clinical judgments and microscopic diagnosis has contributed to the control of oral cancers (17).

In vivo staining reveals cytological details that might otherwise not be apparent. However, staining can also reveal where certain chemicals or specific chemical reactions are taking place within cells or tissues and thus aid in accelerating biopsies, diagnosis and treatment (18). The vital staining methods were used at first in medicine for detecting cervical dysplasia and carcinoma in situ in the 1960s (19). Niebel and Chomet were the pioneers who used dye material to detect oral cancer in 1964 (20). Toluidine blue dye is known as one of the diagnostic adjuncts to detect oral cancer/precancerous lesions. The efficacy of this technique has been evaluated in many reports with diverse results. It has yielded sensitivities between $72 \%$ and $100 \%$, and specificities between $45 \%$ and $67 \%$, in detecting suspicious malignancies (510). However, the Material Data Safety Sheet indicates that toluidine blue is probably toxic by ingestion, and it is seldom used for detecting cancers in other parts of the human body (21). Methylene blue is another recently proposed dye for in vivo staining used in endoscopic examination. Its application has been reported recently in detecting some gastrointestinal abnormalities such as Barrett's esophagus (22-24) gastric cancer (25) prostate cancers $(11,12)$ and also bladder cancer (26). The exact mechanism for the uptake of methylene blue dye in epithelial cells is still not very clear. However, it resembles toluidine blue dye in its acidophilic characteristics. Methylene blue may penetrate into cells with an abnormal increase in nucleic acid, thus resulting in different uptake between normal and highly dysplastic/malignant cells. Usage of the methylene blue technique in detecting oral malignant or potentially malignant lesions has been reported only twice thus far $(4,27)$. This study therefore aimed to study the staining pattern/dye uptake pattern of methylene blue in oral potentially malignant and malignant lesions.

In this study, the usefulness, reliability and shortcomings of methylene blue dye uptake in tissues that have undergone malignant or dysplastic changes have been studied. Possible advantages of methylene blue staining in high risk population have also been detected. The study was 
conducted on high risk patients with unconfirmed mucosal lesions, in a prospective trial to test its diagnostic accuracy in the early detection of potentially malignant and malignant lesions, the staining pattern of the dye and with emphasis on sensitivity, specificity and the significant levels.

In the study the lesions showing dark uptake consisted of 39 cases of non-homogenous leukoplakia, 5 cases of OSCC and 4 cases of erythroplakia. All the cases which showed light uptake were homogenous leukoplakia. A significant difference was observed in the staining pattern and intensities in methylene blue dye uptake in homogenous and non-homogenous leukoplakia due to the difference in the activity of cells in both stages of the disease.

Out of the 25 cases of homogenous leukoplakia which were diagnosed clinically, only 12 cases stained positively for methylene blue dye, and 15 cases were negative for methylene blue stain. Of these, 15 cases which did not stain positively for methylene blue dye, 12 did not show any dysplastic features on histopathology. These 12 cases were true negatives. Three of the 15 cases which showed negative staining showed dysplastic features on histopathology. These were the false negatives. The false negative uptake of this study may be related to areas of interspersed keratin or normal mucosa that did not pick up the stain as keratin does not allow stain penetration. Necrotic portions and deeper extension into tissue planes, of extensive or asymptomatic carcinomas may also not stain for the same reason as mentioned above. As for false-negatives, we consider that the ambiguous light blue stains may be misinterpreted as negative but clinically suspicious of malignancy still need further biopsy to prove the diagnosis pathologically.

In this study, it was also seen that of the 39 cases diagnosed clinically as non-homogenous leukoplakia, all the 39 cases stained positively for methylene blue dye and 34 of the 39 cases showed dysplastic features or were OSCC on histopathological examination. Five cases which stained positively for methylene blue stain clinically but did not show any features of dysplasia pathologically were the 5 false positives of this study. These false positives may be because although, normal tissues should not absorb stain. However, small areas of intense stain may be mechanically retained. Larger areas of excess stain may accumulate on the dorsum of the tongue coated with surface debris and gingival crevices. Occasionally, a light blue film may be observed over a large area of the mucosa as a result of saliva tinged with the dye. The high false positive rate may also be related to the retention of stain in inflamed and trauma areas (8). Other causative factors may include the irregular, papillary or digital surfaces of the lesions, which may cause the mechanical retention of dye, contamination of saliva and plaque, retention of dye material in papilla of the tongue or minor salivary gland ducts over the mucosa. Verrucous hyperplasia and carcinoma may also show mechanical retention of the dye.

Among all the statistical values, sensitivity rate and false negatives were the most important in evaluating the efficacy of certain diagnostic tools for detecting abnormal lesions. Sensitivity represents the proportion of histologically proved malignant/potentially malignant lesions which are detected by positive methylene blue staining. In this study, 55 of the 58 pathologically proved malignant/potentially malignant lesions were positive with deep and focal methylene blue staining. The overall sensitivity of $95 \%$ (100\% for malignancy and $92 \%$ for potentially malignant lesions) was reported, compared to the $72-100 \%$ sensitivity reported in previous studies (5-10). The sensitivity values of this study indicate that using methylene blue dye in the detection of potentially malignant and malignant lesions is acceptable. In the aspect of specificity, we obtained a value $70 \%(12 / 15)$ with a resulting false positive rate of $30 \%$. These results are in accordance to previous study which reported specificity rate of $69 \%$ (21). The lower specificity rate of the present study indicates that not all the lesions which show dye uptake clinically were found to be dysplastic or malignant lesion pathologically. Therefore, biopsy remains the gold standard for diagnosis of oral mucosal lesions.

In our study, we had a positive predictive value of $91 \%$ which meant that 55 out of the 60 cases which stained positively for methylene blue dye correlated well with the pathologic diagnosis of dysplasia. This is much higher than the previously reported study which had a positive predictive value of $74 \%$ (21).

The negative predictive value in our study was $80 \%$ which meant that 12 of the 15 cases 
which did not stain with the methylene blue dye could also be correlated pathologically which showed no evidence of dysplasia. This is in line to previous study which reported specificity rate of $87 \%(21)$.

In conclusion, this study showed that methylene blue staining has nearly $95 \%$ sensitivity in detecting oral malignant or potentially malignant lesions. Considering its low toxicity and the fact that it is cheaper than toluidine blue, it may be convenient to substitute it for toluidine blue in large-scale oral screening of high-risk patients. Nevertheless, the pathology report from biopsy is still the gold standard to accurately diagnose the lesion before a treatment modality is determined.

\section{REFERENCES}

1. Control of oral cancer in developing countries; Bullin WHO. 1984; 62(6):817-30.

2. Shastri SS. Preventive Oncology: Tata Memorial Hospital.

3. Ries LAG, Melbert D, Krapcho M, Stinchcomb DG, Howlader N, Horner MJ, Mariotto A, Miller BA, Feuer EJ, Altekruse SF, Lewis DR, Clegg L, Eisner MP, Reichman M, Edwards BK (2999) SEER cancer statistics review, 1975-2005. National Cancer Institute, Bethesda, $\mathrm{MD}$, http://seer.cancer.gov/csr/1975_ 2005/, based on November 2007 SEER data submission, posted to the SEER web site, 2008 [database online]. 2008. Updated 2008.

4. Ya-Wei C. Application of in vivo stain of methylene blue as a diagnostic aid in the early detection and screening of oral squamous cell carcinoma and precancerous lesion. J Chin Med Assoc. 2007;70:497-503.

5. Epstein JB, Scully C, Spinelli J. Toluidine blue and Lugol's iodine application in the assessment of oral malignant disease and lesions at risk of malignancy. J Oral Pathol Med. 1992; 21:160-163.

6. Epstein JB, Oakley C, Millner A, Emerton S, van der Meij E. The utility of toluidine blue as $\mathrm{s}$ a diagnostic aid in patients previously treated for upper oropharyngeal carcinoma. Oral Surg Oral Med Oral Pathol Oral Radiol Endod. 1997; 83(5):537-547.
7. Mashberg A. Re-evaluation of toluidine blue application as a diagnostic adjunct in the detection of asymptomatic oral squamous carcinoma. Cancer, 1980;46:758-763.

8. Mashberg A. Final evaluation of tolonium chloride rinse for screening of highrisk patients with asymptomatic squamous carcinoma. JADA, 1983;106:319-323.

9. Onofre MA, Sposto MR, Navarro CM. Reliability of toluidine blue application in the detection of oral epithelial dysplasia and in situ and invasive squamous cell carcinomas. Oral Surg Oral Med Oral Pat Oral Radiol Endod, 2001;91:535-540.

10. Warnakulasuriya KA, Johnson NW Sensitivity and specificity of OraScan toluidine blue mouthrinse in the detection of oral cancer and precancer. J Oral Pathol Med, 1996; 25:97103.

11. Mufti GR, Shah PJ, Parkinson MC, Riddle PR. Diagnosis of clinically occult bladder cancer by in vivo staining with methylene blue. Br J Urol, 1990;65:173-175.

12. Gill WB, Huffman JL, Lyon ES, Bagley DH, Schoenberg HW, Straus FH $2^{\text {nd }}$. Selective surface staining of bladder tumors by intravesical methylene blue with enhanced endoscopic identification. Cancer, 1984;53:2724-2727.

13. Yu DS, Chang SY, Ma CP. Photoinactivation of bladder tumor cells by methylene blue: study of a variety of tumor and normal cells. $J$ Urol, 190;144(1):164-168.

14. Kuo MY, Jeng JH, Chiang CP, Hahn LJ. Mutations of Ki-ras oncogene codon 12 in betel quid chewing-related human oral squamous cell carcinoma in Taiwan. J Oral Pathol Med, 1994;23(2):70-4.

15. Reibel J. Prognosis of oral premalignant lesions: Significance of clinical histopathological, and molecular biological characteristics. Crit Rev Oral Biol Med, 2003; 14(1): 47-62.

16. Martin IC, Kerawala CJ, Reed M. The application of toluidine blue as a diagnostic adjunct in the detection of epithelial dysplasia. Oral Surg Oral Med Oral Pathol Oral Radiol Endod, 1998;85(4):444-6.

17. Saku T, Okabe H. Differential lectin-bindings in normal and precancerous epithelium and 
squamous cell carcinoma of the oral mucosa. $J$ Oral Pathol Med. 1989;18(8):438-45.

18. Clark G Staining Procedures, 1981. 4th ed, 412. Baltimore: Williams \& Wilkins.

19. Richart RM. Clinical staining test for the in vivo delineation of dysplasia and carcinoma in situ. Am J Obstet Gynecol. 1963; 15; 86:70312.

20. Niebel $\mathrm{HH}$, Chomet B. In vivo staining for delineation of oral intraepithelial neoplastic change. Preliminary report. J Am Dent Assoc. 1964; 68:801-6.

21. Ya-Wei Chen, Jiun-Sheng Lin, Jenny HwaiJen Fong, I-Kai Wang, Shen-Ju Chou,ChenHsian Wu, Man-Tin Lui, Che-Shoa Chang, Shou-Yen Kao.Use of methylene blue as a diagnostic aid in early detection of oral cancer and precancerous lesions. British Journal of Oral and Maxillofacial Surgery, 2007;45:590591.

22. Canto MI, Setrakian S, Willis JE, Chak A, Petras RE, Sivak MV. Methylene blue staining of dysplastic and nondysplastic Barrett's esophagus: an in vivo and ex vivo study. Endoscopy. 2001; 33(5):391-400.
23. Canto MI, Setrakian S, Willis J, Chak A, Petras R, Powe NR, Sivak MV Jr. Methylene blue-directed biopsies improve detection of intestinal metaplasia and dysplasia in Barrett's esophagus. Gastrointest Endosc. 2000; 51(5):560-8.

24. Canto MI, Setrakian S, Petras RE, Blades E, Chak A, Sivak MV Jr. Methylene blue selectively stains intestinal metaplasia in Barrett's esophagus. Gastrointest Endosc. 1996; 44(1):1-7.

25. Suzuki S, Murakami H, Suzuki H, Sakakibara $\mathrm{N}$, Endo M, Nakayama K. An endoscopic staining method for detection and operation of early gastric cancer. Int Adv Surg Oncol, $1979 ; 2: 223-41$.

26. Fukui I, Yokokawa M, Mitani G, Ohwada F, Wakui M, Washizuka M, Tohma T, et al.In vivo staining test with methylene blue for bladder cancer. J Urol, 1983; 130:252-255.

27. Riaz A, Shreedhar B, Kamboj M, Natarajan S. Methylene blue as an early diagnostic marker for oral precancer and cancer. Springerplus. 2013;2(1):95. 